\title{
Time-averaged intensity spectral density of acoustic radiation due to time-varied lift force of rotating blades
}

\author{
Kiyoto Ishimaru \\ Hamamatsu Polytechnic College \\ 693 Norieda-cho, Hamamatsu, 432 Japan
}

(Received 9 November 1988)

\begin{abstract}
The time-averaged intensity spectral density function of the acoustic radiation from rotating blades is theoretically derived by replacing the blades with rotating dipoles. Dimensional analysis reveals two non-dimensional parameters which play an important role in generating the blade-passing frequency tone and its multiples. Increasing the number of rotor blades widens the peak under the condition that the non-dimensional parameter of the rotational speed is fixed. Moreover, the acoustic spectral density function is not stationary even if the inflow turbulence is homogeneous and isotropic. Further, the time variation of the propagation path due to the rotation should be considered in the computation of the spectral density function. For instance, in the rotor specifications of the present study, the rotor radius is approximately $0.3 \mathrm{~m}$ and the rotational speed Mach number is approximately 0.2 .
\end{abstract}

PACS number: 43. 28. Ra

\section{INTRODUCTION}

This paper attempts to derive the time-averaged farfield acoustic intensity spectral density function due to the acoustic radiation from low rotational speed rotors starting with Lighthill's acoustic analogy approach, where the rotor blade is assumed to be acoustically compact. For the present analysis, the dipole source due to blade/turbulence interaction is assumed to be predominant over other radiation sources. A rotating dipole model is examined so that the effect of the dipole rotation can be explicitly accounted for in the analysis. Furthermore, there is no blade-to-blade interaction to be included, such as that developed by Kemp et al. ${ }^{1)}$ The assumption of acoustically compact blades permits replacing distributed dipole sources with a single-point dipole whose strength is equal to the distributed dipoles on the blade. Thus, each blade is replaced with a point dipole or multiples of a dipole.

Lowson $^{2)}$ derived the far-field and near-field acoustic pressure from a moving singular dipole source by using Lighthill's acoustic analogy approach, where the importance of the acceleration of the moving dipole was accounted for in the expression of acoustic pressure, while the dipole strength was assumed to be constant. Sevik ${ }^{3)}$ derived the sound power spectrum from a rotor operating in turbulence. The noise source is the axial component of the blade lift only. The sound power $S_{\mathrm{p}}(\omega)$ is computed based on the general relation $S_{\mathrm{p}}(\omega)=$ $E\left\{|P(\omega)|^{2}\right\}$, where $S_{\mathrm{p}}(\omega)$ is the power spectrum of acoustic pressure $p(t)$ satisfying $\int_{-\infty}^{\infty} \alpha R_{\mathrm{pp}}(\alpha) d \alpha<\infty$, where $R_{\mathrm{pp}}(\alpha)$ is the correlation function of $p(t)$ which is assumed to be a wide-sense-stationary process, and the correlation function regards the rotor as being stationary. However, because of the use of only the axial component of the lift, the result applies only near the center line axis of the rotor. Unfortunately, the resulting power spectrum does not predict the blade-passing frequency component 
and its multiples, due to the assumption of the correlation function of the upwash at the blades, although, as shown by Robbins et al.,4) the level and general shape of the predicted spectra agree with the experimental data.

This paper attempts to introduce moving dipoles with a time-variant strength into the theory of Lowson $^{2)}$ to consider more practical situations, and to relate the acoustic intensity spectral density to the spectrum of the blade lift taking into account the rotation of the blade to extend Sevik's theory. In addition, discussed is the influence on the spectrum of the time variation of the propagation path due to the rotation.

\section{FAR-FIELD ACOUSTIC PRESSURE}

The analysis of the acoustic radiation from rotating blades begins with the nonhomogeneous acoustic radiation from the boundary condition imposed by the blades. The Lighthill acoustic analogy approach leads to an integral equation, with Green's function, for an infinite medium where the integration is carried out over three spatial dimensions and one temporal dimension. Here, the time is regarded as a parameter; i.e., the so-called retarded time concept is employed. It is assumed from the discussion of Farassat ${ }^{5)}$ that the surface of a moving body can be replaced with a point dipole source whose strength is equal to the total force on the virtual radiation surface providing the following relation is satisfied:

$$
\tau_{\mathrm{p}} \ll L / a
$$

where $\tau_{\mathrm{p}}$, and $L$ are the characteristic time scale of the surface pressure, and the length scale of the virtual surface of an acoustical source.

Thus, the acoustic wave equation to be solved for rotating blades is

$$
\nabla^{2} \mathscr{Q}-\frac{1}{a^{2}} \frac{\partial_{2} \mathscr{Q}}{\partial \tau^{2}}=-\nabla \cdot \vec{f}
$$

where

$$
\vec{f}=\sum_{m=1}^{B} \sum_{n=1}^{N} \vec{l}_{m n}(\tau) \delta\left(\Phi-\Omega \tau-\Phi_{m}\right) \delta(Z) \delta\left(R-R_{n}\right) \frac{\Delta R_{n}}{R}
$$

Figure 1 shows the configuration of the relation between the rotating dipole and the observation point, where spherical coordinates and cylindrical coordinates are used for the observation point and the rotating dipole, respectively.

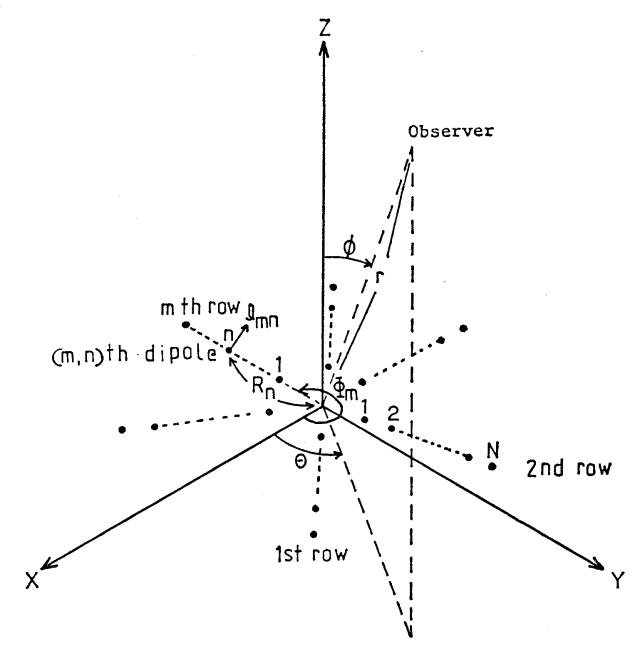

Fig. 1 Observer/source coordinates.

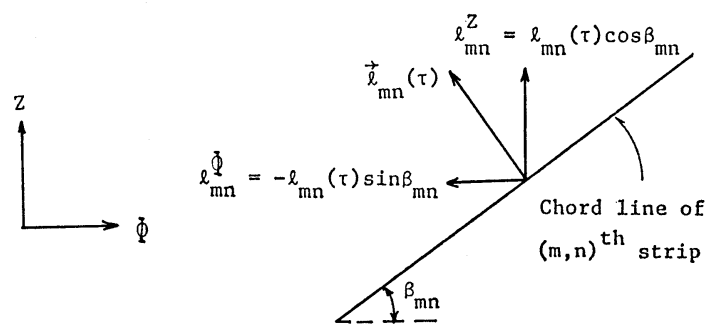

Fig. 2 Lift vector components at $(m, n)$ th strip.

With the aid of the fundamental solution of Eq. (2.2), imposing the conditions for the acoustic farfield and geometrical far-field, and introducing $l_{m n}^{\Phi}(\tau)=-\left|\vec{l}_{m n}(\tau)\right| \sin \beta_{m n}$ and $l_{m n}^{Z}(\tau)=\left|\vec{l}_{m n}(\tau)\right| \cos \beta_{m n}$ where $\vec{l}_{m n}(\tau)$ is assumed to be normal to the chord line of a thin airfoil (see Fig. 2), then the following equation is obtaine,

$$
\begin{aligned}
\mathcal{L}(r, \phi, t) & \frac{1}{4 \pi a r}\left(\sum_{m=1}^{B} \sum_{n=1}^{N} \frac{\Delta R_{n}}{\left(1+\frac{R_{n} \Omega}{a} \sin \phi \sin \left(\Omega \tau+\Phi_{m}\right)\right)^{2}}\right. \\
& \times\left\{-\frac{\partial l_{m n}}{\partial \tau} \sin \beta_{m n} \sin \phi \sin \left(\Omega \tau+\Phi_{m}\right)\right. \\
& -\frac{\partial l_{m n}}{\partial \tau} \cos \beta_{m n} \cos \phi-l_{m n} \Omega \sin \beta_{m n} \sin \phi \\
& \times \frac{\cos \left(\Omega \tau+\Phi_{m}\right)}{1+\frac{R_{n} \Omega}{a} \sin \phi \sin \left(\Omega \tau+\Phi_{m}\right)}
\end{aligned}
$$




\section{K. ISHIMARU: NOISE SPECTRUM FROM ROTATING BLADES}

$$
\begin{aligned}
& +l_{m n} \Omega \cos \beta_{m n} \cos \phi \sin \phi \\
& \left.\left.\times \frac{\frac{R_{n} \Omega}{a} \cos \left(\Omega \tau+\Phi_{m}\right)}{1+\frac{R_{n} \Omega}{a} \sin \phi \sin \left(\Omega \tau+\Phi_{m}\right)}\right\}\right):
\end{aligned}
$$

where

$$
\tau \approx t-D_{m n} / a,
$$

and

$$
D_{m n}=r-R_{n} \sin \phi \cos \left(\Omega \tau+\Phi_{m}\right) .
$$

Equations (2.4) is an extension of the results of Lowson $^{2)}$ and Morfey et al., ${ }^{6)}$ which deal with the acoustic radiation from a moving point force. As will be shown, this relates the acoustic intensity spectral density function at the observation point with the power spectral density function of the turbulence experienced by rotating blades.

As shown in Eq. (2.4), the variation of the distance between the observer and the blade on a time axis causes a phase modulation in $l_{m n}(\tau)$ because $\tau \approx t-\left(r-R_{n} \sin \phi \cos \left(\Omega \tau+\Phi_{m}\right)\right) / a$ where the numerator is the distance between the observer and the blade. Furthermore, the variation of the blade velocity Mach number in the direction of the observer, i.e., $\left(R_{n} \Omega / a\right) \sin \phi \sin \left(\Omega \tau+\Phi_{m}\right)$, behaves as an amplitude modulation signal of $l_{m n}(\tau)$ and $\partial l_{m n}(\tau) / \partial \tau$.

Utilizing the results shown in the Appendix, Eq. (2.4) can be rewritten as follows:

$$
\begin{aligned}
\mathscr{Q}(r, \phi, t)= & \frac{1}{4 \pi a r} \sum_{m=1}^{B} \sum_{n=1}^{N} \sum_{p=-\infty}^{\infty}\left(e_{m n}^{(p)} \frac{\partial l_{m n}}{\partial \tau}-j \Omega f_{m n}^{(p)} l_{m n}\right) \\
& \times \exp \left[j p\left(\Omega t+\Phi_{m}+\frac{\pi}{2}\right)\right],
\end{aligned}
$$

where

$$
\begin{gathered}
e_{m n}^{(p)}=-b_{n}^{(p)^{\prime}} \sin \phi \sin \beta_{m n}-a_{n}^{(p)^{\prime}} \cos \phi \cos \beta_{m n} \\
f_{n n}^{(p)}=\sin \phi\left(\cos \phi \cos \beta_{m n}-\sin \beta_{m n}\right) c_{n}^{(p)^{\prime}} \\
c_{n}^{(p)^{\prime}}=\sum_{p=-\infty}^{\infty} \frac{p J_{p}\left(p \frac{R_{n} \Omega}{a} \sin \phi\right)}{\frac{R_{n} \Omega}{a} \sin \phi} \\
a_{n}^{(p)^{\prime}}=\sum_{s=-\infty}^{\infty} \sum_{k=-\infty}^{\infty} J_{s}\left(s \frac{R_{n} \Omega}{a} \sin \phi\right) J_{k}\left(k \frac{R_{n} \Omega}{a} \sin \phi\right) \\
p=s+k,
\end{gathered}
$$

and

$$
b_{n}^{(p)^{\prime}}=\sum_{s=-\infty}^{\infty} \sum_{k=-\infty}^{\infty} \frac{\delta_{\mathrm{os}}-1}{\frac{R_{n} \Omega}{a} \sin \phi}
$$

$$
\begin{gathered}
\times J_{s}\left(s \frac{R_{n} \Omega}{a} \sin \phi\right) J_{k}\left(k \frac{R_{n} \Omega}{a} \sin \phi\right) \\
p=s+k .
\end{gathered}
$$

The term, $e^{j p \Omega \tau}$, represents the explicit expression of the time variation of radiation path on a source time axis under the assumption that the typical time scale of $l_{m n}(\tau)$ is very large compared with $R_{n} / a$.

In Eq. (2.5), the second term dominates over the first term when $l_{m n}(\tau)$ is constant, i.e., the variation of the blade velocity Mach number in the direction of the observer gives rise to the blade-passing frequency and its multiples, and conversaly the first term dominates over the second when $\phi=0$, i.e., there is no variation of the blade velocity in the direction of the observer. Hence, the second term is strongly related to the blade acceleration. Accordingly, a model which does not take the dipole acceleration into consideration such as the rectilinear model, is applicable only where $\Omega \ll \omega$, since the first term dominates over the second term when $\Omega \ll \omega$.

Equation (2.5) reveals that even when $l_{m n}(\tau)$ is statistically stationary the resulted acoustical pressure is not so due to the term $e^{j p 2 t}$. This is the reason why taking the time average over $2 \pi / \Omega$ is done in the next section to get rid of the time-dependency of the intensity spectrum. Furthermore, the observer even at $\phi=0$, i.e., $R_{n} \Omega \sin \phi / a \ll 1$, has a possibility to hear the blade-passing frequency and its multiples depending on the inflow condition, although the variation of the blade velocity in the direction of the observer can be neglected even when $R_{n} \Omega \sin \phi / a \ll 1$. This is to be shown in the numerical results of the later section.

\section{TIME-AVERAGED INTENSITY SPECTRAL DENSITY FUNCTION}

Utilizing the following definition of the autocorrelation function of $\mathcal{Q}(r, \phi, t)$,

$$
\mathscr{R}_{\mathscr{Q} \mathscr{P}}(r, \phi, t, \alpha)=E\left\{\mathscr{L}^{*}(r, \phi, t) \mathscr{L}(r, \phi, t+\alpha)\right\},
$$

and the following pair of Fourier integrals,

$$
\begin{aligned}
& S_{\mathscr{Q} \mathscr{P}}(r, \phi, t, \omega) \\
& \quad=(1 / 2 \rho a) \int_{\infty}^{-\infty} \mathscr{R}_{\mathscr{Q} \mathscr{P}}(r, \phi, t, \alpha) e^{-j \omega \alpha} d \alpha
\end{aligned}
$$

and

$$
\begin{aligned}
& \mathscr{R}_{\mathscr{Q} \mathscr{Q}}(r, \phi, t, \alpha) \\
& \quad=(\rho a / \pi) \int_{\infty}^{-\infty} S_{\mathscr{Q} \mathscr{P}}(r, \phi, t, \omega) e^{j \omega \alpha} d \omega,
\end{aligned}
$$


the expected value of the acoustic intensity at a large distance $r$ is given by

$$
E\left\{I_{r}(r, \phi, t)\right\}=(1 / 2 \pi) \int_{\infty}^{-\infty} S_{\mathscr{Q} \mathscr{P}}(r, \phi, t, \omega) d \omega
$$

Thus, $S_{\mathscr{P} \mathcal{P}}(r, \phi, t, \omega)$ can be regarded as an instantaneous intensity spectral density function.

Assuming $S_{\mathscr{P} \mathcal{P}}(r, \phi, t, \omega)$ is continuous and finite with respect to $t$, the typical time scale of $l_{m n}(\tau)$ is very large compared with $R_{n} / a$ (see Eq. (2.1)), $l_{m n}(\tau)$ is statistically stationary, replacing a blade with a dipole located at $R=R_{\mathrm{T}}$, introducing a typical impulse response function $h(t)$ of a blade for all $\mathrm{m}$ such that

$$
l_{m n}(\tau)=\int_{-\infty}^{\infty} h(\tau-\eta) u_{m}(\eta) d \eta
$$

where $u_{m}(t)$ is the upwash at the $m$ th blade, and timeaveraging $S_{\mathscr{Q} \mathscr{P}}(r, \phi, t, \omega)$ over $2 \pi / \Omega$, the time-averaged intensity spectral density function can be obtained as follows

$$
\begin{aligned}
\left\langle S_{\mathscr{Q} \mathscr{P}}(r, \phi, t, \omega)\right\rangle \\
\left.=\left\{\left(\Delta R_{\mathrm{T}}\right)^{2}\right\} /\left(32 \rho a^{3} \pi^{2} r^{2}\right)\right\} \sum_{p=-\infty}^{\infty}|H(\omega-p \Omega)|^{2} \\
\quad \times\left\{(\omega-p \Omega)^{2}\left(e_{\mathrm{T}}^{(p)}\right)^{2}+\Omega^{2}\left(f_{\mathrm{T}}^{(p)}\right)^{2}\right\} \\
\quad \times\left\{\sum_{m=1}^{B} \sum_{k=1}^{B} \exp \{j(2 \pi / B) p(k-m)\} S_{m k}^{\mathrm{uu}}(\omega-p \Omega) .\right.
\end{aligned}
$$

In Eq. (3.6), $S_{m k}^{\mathrm{uu}}(\omega)$ is the Fourier transformed correlation function between $u_{m}(t)$ and $u_{k}(t)$ assuming the upwash is stationary, and $H(\omega)$ is the Fourier transformed impulse function, i.e., a transfer function. Equation (3.6) reveals the relation among the transfer function of a blade $H(\omega)$, the time variation of the propagation path $p$, the statistical property of the inflow $S_{m k}^{\mathrm{uu}}(\omega)$, and the spatial relation among blades $e^{j[2 \pi(k-m)] / B}$.

Letting the transfer function be the approximate form of Sears' function ${ }^{7,8)}$ such that

$$
\begin{aligned}
& H(\omega) \simeq \pi \rho \Omega c_{\mathrm{T}} \\
& \quad \times \frac{\exp \left\{-j\left(\omega c_{\mathrm{T}} / 2 R_{\mathrm{T}} \Omega\right)\left(1-\pi^{2} / 2\left(1+\pi \omega c_{\mathrm{T}} / R_{\mathrm{T}} \Omega\right)\right)\right\}}{\left(1+\pi \omega c_{\mathrm{T}} / R_{\mathrm{T}} \Omega\right)^{1 / 2}},
\end{aligned}
$$

utilizing the result of Lane ${ }^{9)}$ with respect to the correlation function, $\mathscr{R}_{m k}^{\mathrm{uu}}(\alpha)$, and non-dimensionalizing Eq. (3.6), Eq. (3.6) becomes

$$
\begin{aligned}
& \frac{\left\langle S_{\mathscr{Q} \mathcal{Q}}(r, \phi, t, \omega)\right\rangle}{\rho a^{2} R_{\mathrm{T}}} \\
& =(1 / 32)\left(c_{\mathrm{T}} / r\right)^{2}\left(\Delta R_{\mathrm{T}} / R_{\mathrm{T}}\right)^{2}\left(R_{\mathrm{T}} \Omega / a\right)^{3}\left(\overline{\left.\left(\overline{u^{2}}\right) / a^{2}\right)}\right.
\end{aligned}
$$

$$
\begin{aligned}
& \times \sum_{p=-\infty}^{\infty}\left\{\frac{\{(\omega-p \Omega) / \Omega\}^{2}\left(e_{\mathrm{T}}^{(p)}\right)^{2}+\left(f_{\mathrm{T}}^{(p)}\right)^{2}}{\pi|(\omega-p \Omega) / \Omega|\left(c_{\mathrm{T}} / R_{\mathrm{T}}\right)+1}\right. \\
& \left.\times S_{B}\left(U_{z} / \Lambda_{\mathrm{i}} \Omega, R_{\mathrm{T}} / \Lambda_{\mathrm{i}},(\omega-p \Omega) / \Omega\right)\right\}
\end{aligned}
$$

where

$$
\begin{aligned}
& S_{B}\left(U_{z} / \Lambda_{\mathrm{i}}, R_{\mathrm{T}} / \Lambda_{\mathrm{i}}, \omega\right)=\sum_{m=1}^{B} \sum_{k=1}^{B} \exp \{j(2 \pi / B) p(k-m)\} \\
& \times S\left(U_{z} / \Lambda_{\mathrm{i}} \Omega, R_{\mathrm{T}} / \Lambda_{\mathrm{i}}, \omega\right), \\
& \begin{aligned}
S\left(U_{z} / \Lambda_{\mathrm{i}} \Omega, R_{\mathrm{T}} / \Lambda_{\mathrm{i}}, \omega\right)=\left(\Omega / \overline{u^{2}}\right) & S_{k k}^{\mathrm{uu}}(\omega),
\end{aligned} \\
& S_{m k}^{\mathrm{uu}}(\omega)=\int_{-\infty}^{\infty} \mathcal{R}_{m k}^{\mathrm{uu}}(\alpha) e^{-j \omega \alpha} d \alpha, \\
& \mathcal{R}_{m k}^{\mathrm{uu}}(\alpha)=\bar{u}^{2}\{E(\alpha)(1-G(\alpha) / 2)+F(\alpha) / 2 G(\alpha)\} e^{-G(\alpha)}, \\
& E(\alpha)=\cos ^{2} \beta+\cos (\Omega \alpha+2 \pi(k-m) / B) \sin ^{2} \beta, \\
& G^{2}(\alpha)=\left(U_{z} \alpha / \Lambda_{\mathrm{i}}\right)^{2} \\
& \quad+2\left(R_{\mathrm{T}} / \Lambda_{\mathrm{i}}\right)^{2}\{1-\cos (\Omega \alpha+2 \pi(k-m) / B)\},
\end{aligned}
$$

and

$$
\begin{aligned}
F(\alpha)= & \left(U_{z} \alpha / \Lambda_{\mathrm{i}}\right)^{2} \cos ^{2} \beta \\
& +\left(R_{\mathrm{T}} / \Lambda_{\mathrm{i}}\right)^{2} \sin ^{2} \beta \sin ^{2}(\Omega \alpha+2 \pi(k-m) / B) .
\end{aligned}
$$

The terms $E(\alpha)$ and $F(\alpha)$ in the above equation are introduced to make the equation shorten, and $G(\alpha)$ is the distance between two physical points in the converted turbulence with which the blades interact. Hence, $G(\alpha)$ is a function of time. In the derivation of $\mathscr{R}_{m k}^{\mathrm{uu}}(\alpha)$, utilized are the assumptions: (1) an

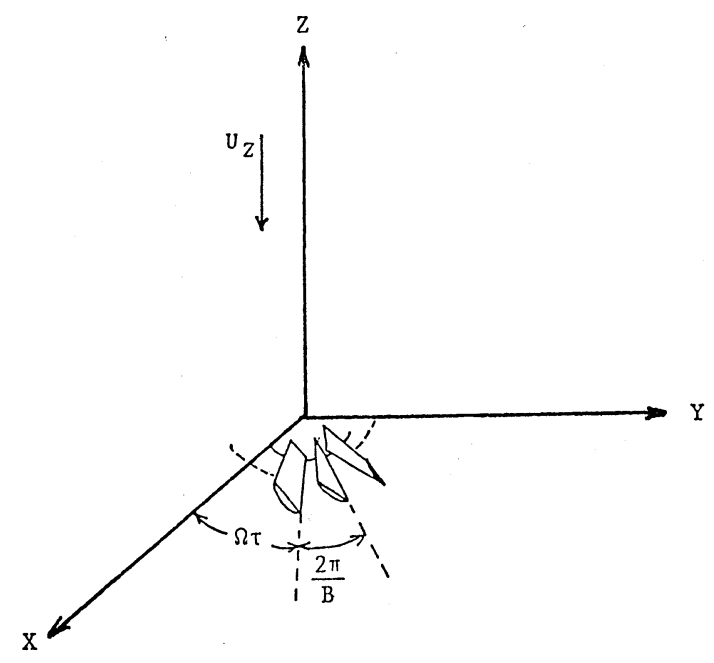

Fig. 3 Rotor in cylindrical coordinates. 


\section{K. ISHIMARU: NOISE SPECTRUM FROM ROTATING BLADES}

incompressible fluid medium, (2) frozen turbulence pattern convected with an axial flow velocity $U$, (3) homogeneous and isotropic turbulence, see Fig. 3, and (4) a longitudinal function, $\exp \left(-G / \Lambda_{\mathrm{i}}\right)$, where $\Lambda_{\mathrm{i}}$ is the integral scale of the turbulence, and $G$ is the distance between two physical points in the turbulence. As revealed in Eq. (3.14), the distance $G$ is a function of time since the distance is the one between two physical points in the frozen turbulence pattern convected with an axial flow to be experienced by rotating blades. Due to homogeneous and isotropic turbulence assumption, the correlation function is stationary.

Utilization of Sears' function imposes that the effect of the flow fluctuation parallel to the chord line can be neglected as a second order effect. Hence, our concern is with the response of a thin airfoil with zero mean angle of attack to an upwash velocity fluctuation, where the airfoil is assumed to be lightly loaded so that the stagger angle $\beta$ can be approximated by $U_{z} / R_{\mathrm{T}} \Omega$. Another important consequence from utilizing Sears' function, under the assumption of the frozen turbulence pattern convected with an axial flow velocity $U_{z}$, is that a one-to-one correspondence between frequency and upwash wavenumber is attained so that the whole noise generation process can be treated on a time and frequency domain instead of a time and spatial domain. In addition, utilization of Sears' function is heavily relied on the results by Jackson et al. ${ }^{10)}$ : the three-dimensional interaction between blades and flow fluctuations can be approximated by a two-dimensional strip theory using Sears' function. The two-dimensional strip approach imposes that the acoustic radiation due to parallel gusts (the spanwise wavenumber is zero) is dominant, as investigated by Amiet. ${ }^{11)}$

Equation (3.8) shows the relation between $\left\langle S_{\mathscr{Q} \mathcal{P}}(r, \phi, t, \omega)\right\rangle$ and the terms: (1) amplitude modulation terms due to rotation, i.e., $e_{\mathrm{T}}^{(p)}$ and $f_{\mathrm{T}}^{(p)}$, (2) the blade transfer function $H(\omega)$, and (3) the upwash-related spectrum $S_{B}\left(U_{z} / \Lambda_{\mathrm{i}} \Omega, R_{\mathrm{T}} / \Lambda_{\mathrm{i}}, \omega / \Omega\right)$. The non-dimensional parameters, $U_{z} / \Lambda_{\mathrm{i}} \Omega$ and $R_{\mathrm{T}} / \Lambda_{\mathrm{i}}$, represent the relations between the turbulence and the rotor, dynamic and kinematic relation, respectively. The dynamic relation implies how many times the blade chops the statistically related turbulence on the course of time passing. On the other hand, the kinematic relation implies how many blades can chop the statistically related turbulence at an instance time. The next section shows how these two non-dimensional parameters govern the profile of $\left\langle S_{\mathscr{Q} \mathscr{Q}}(r, \phi, t, \omega)\right\rangle$.

\section{NUMERICAL RESULTS}

Computations are done for the results in Sections 2 and 3 by changing the number of blades, the observation angle, and non-dimensional parameters such as $U_{z} / \Lambda_{\mathrm{i}} \Omega$ and $R_{\mathrm{T}} / \Lambda_{\mathrm{i}}$. In particular, the variation of these non-dimensional parameters is made around an axial flow velocity of $U_{z}=30 \mathrm{~m} / \mathrm{s}$, a rotor, radius of $R_{\mathrm{T}}=0.30 \mathrm{~m} / \mathrm{s}$, a rotor rotational speed of $\Omega=220 \mathrm{rad} / \mathrm{s}$, an inflow turbulence length scale $\Lambda_{\mathrm{i}}=$ $0.15 \mathrm{~m}$.

Figures 4, 5, and 6 show the non-dimensionalized $\left\langle S_{\mathscr{Q} \mathcal{P}}(r, \phi, t, \omega)\right\rangle$ dependent on the non-dimensional parameters, $U_{z} / \Lambda_{\mathrm{i}} \Omega$ and $R_{\mathrm{T}} / \Lambda_{\mathrm{i}}$. These figures show that as $U_{z} / \Lambda_{\mathrm{i}} \Omega$ decreases, the level of the bladepassing frequency and its multiples increases, and as $R_{\mathrm{T}} / \Lambda_{\mathrm{i}}$ increases those levels also increase. Furthermore, as $R_{\mathrm{T}} / \Lambda_{\mathrm{i}}$ increases, the overall level increases.

Hence, it is clear that the kinematic relation $R_{\mathrm{T}} / \Lambda_{\mathrm{i}}$ cannot explain fully the spectral profile without the dynamic relation $U_{z} / \Lambda_{\mathrm{i}} \Omega$. The effect of $R_{\mathrm{T}} / \Lambda_{\mathrm{i}}$ on the spectrum for constant $U_{z} / \Lambda_{\mathrm{i}} \Omega$ is the opposite of that claimed by Mani. ${ }^{12)}$ This is probably due to the fact that Mani did not consider the variation of $U_{z} / \Lambda_{\mathrm{L}} \Omega$ when he varied $R_{\mathrm{T}} / \Lambda_{\mathrm{I}}$ as a parameter. Further investigation of this matter is necessary.

Furthermore, as the rotational speed increases, the bandwidth and the level at the blade-passing frequency and its multiples are increased, and as the axial flow velocity decreases, the blade-passing frequency and its multiples appear clearly. All these variations, under the constant turbulence length scale constraint, make rotating blades interact with the statistically correlated turbulence more. Hence, the above phenomenon can be observed through the variation of $U_{z} / \Lambda_{1} \Omega$ in Figs. 4, 5, and 6.

Figure 7 shows that as $B$ increases, the bandwidth of the peak at the blade-passing frequency and its multiples increases. In this example, as $B$ is increased from 3 to 9 , the width at the blade-passing frequency has approximately triples. This tendency is mentioned also by Homicz. ${ }^{13)}$ This is probably due to the fact that as $B$ increases the correlation among the noise sources (rotating dipoles) is increased so that the spectral profile is flatten.

Figure 8 shows the observer angle dependence 


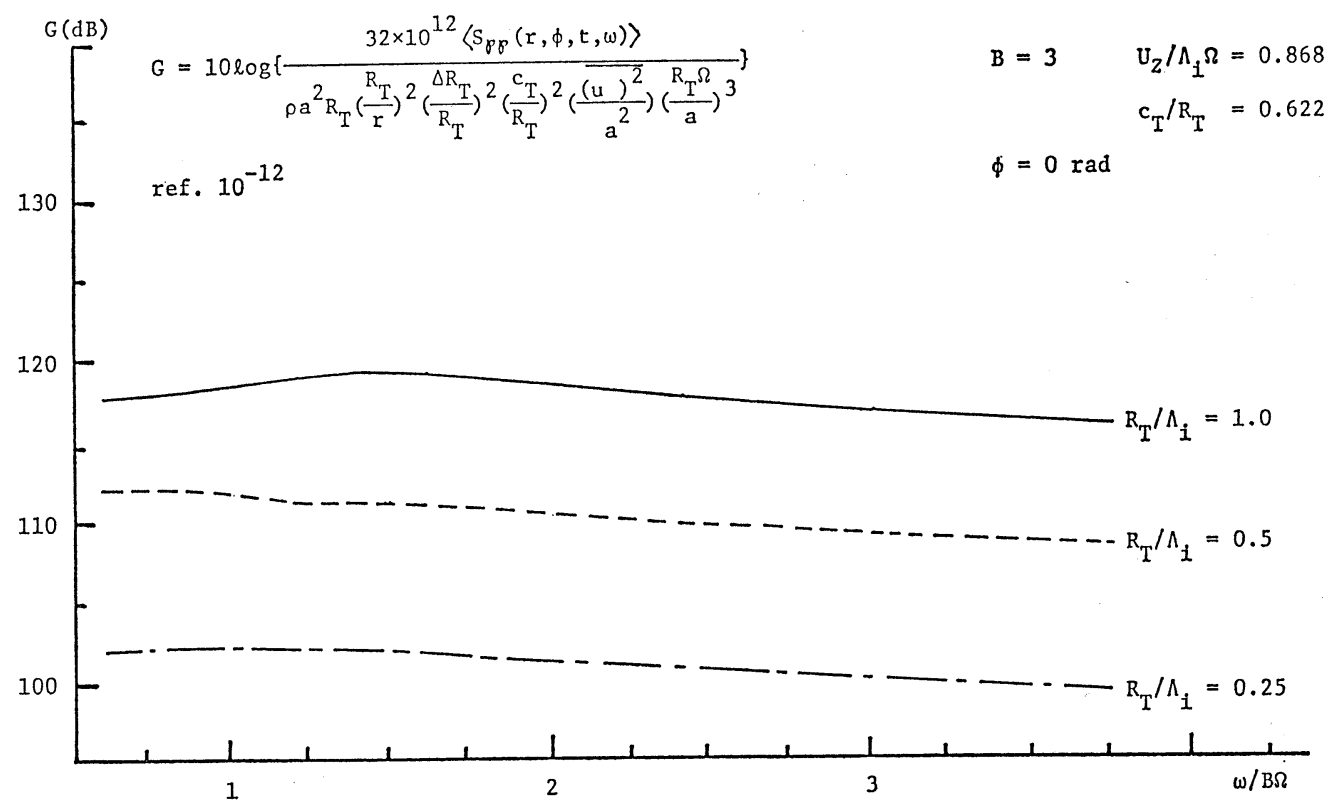

Fig. 4 Non-dimensionalized $\left\langle S_{\mathscr{Q}} \mathscr{Q}(r, \phi, t, \omega)\right\rangle$ for $B=3$ and $U_{z} / \Lambda_{\mathrm{i}} \Omega=0.868$.

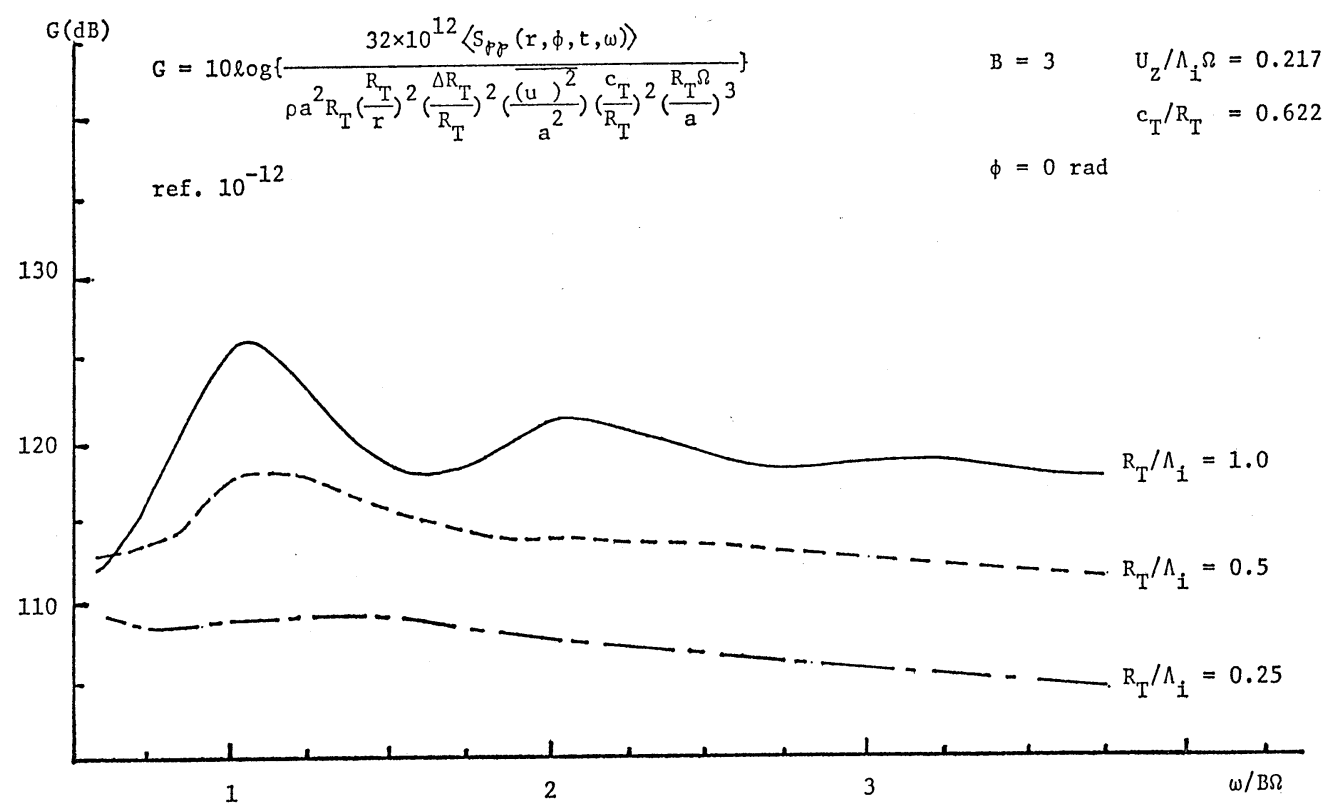

Fig. 5 Non-dimensionalized $\left\langle S_{\mathscr{Q} \mathscr{Q}}(r, \phi, t, \omega)\right\rangle$ for $B=3$ and $U_{z} / \Lambda_{\mathrm{i}} \Omega=0.217$ 


\section{K. ISHIMARU: NOISE SPECTRUM FROM ROTATING BLADES}

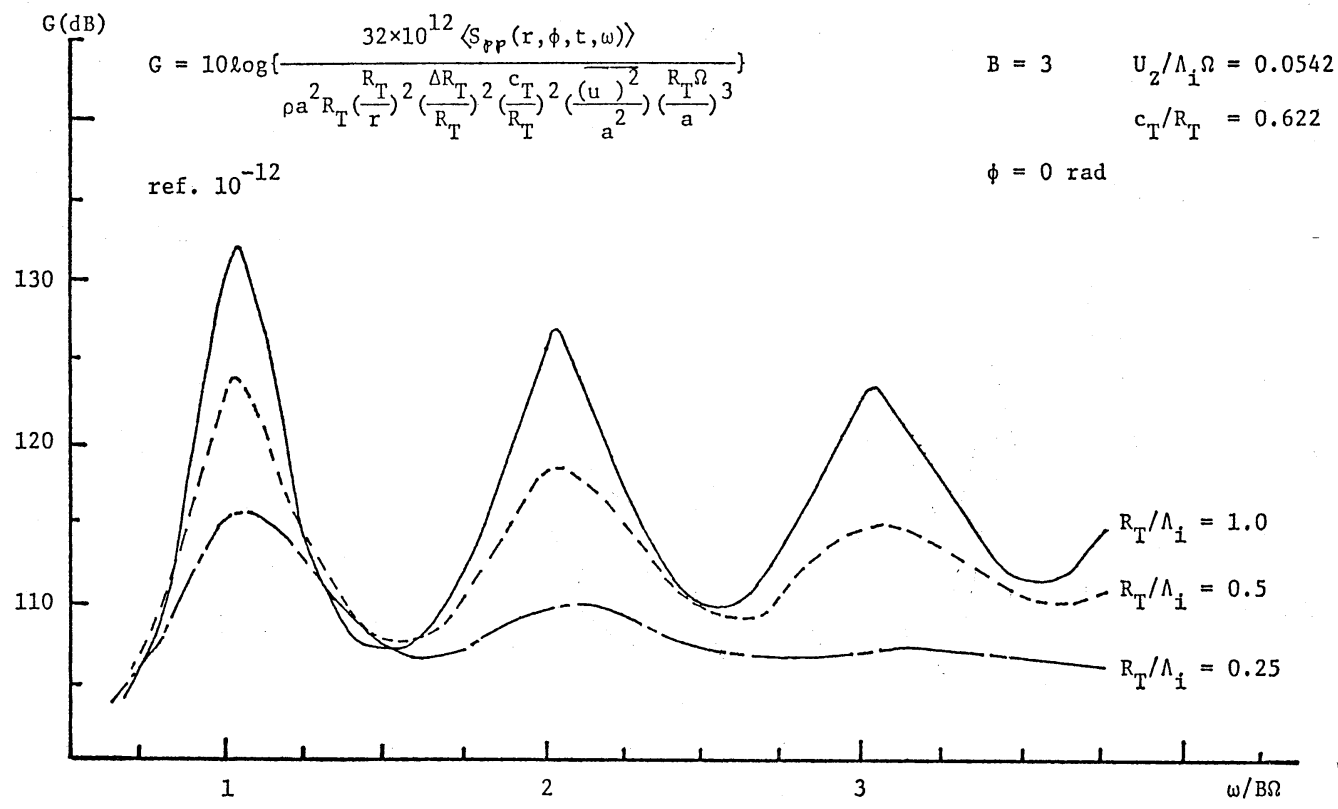

Fig. 6 Non-dimensionalized $\left\langle S_{\mathcal{Q} \mathscr{Q}}(r, \phi, t, \omega)\right\rangle$ for $B=3$ and $U_{z} / \Lambda_{\mathrm{i}} \Omega=0.0542$.

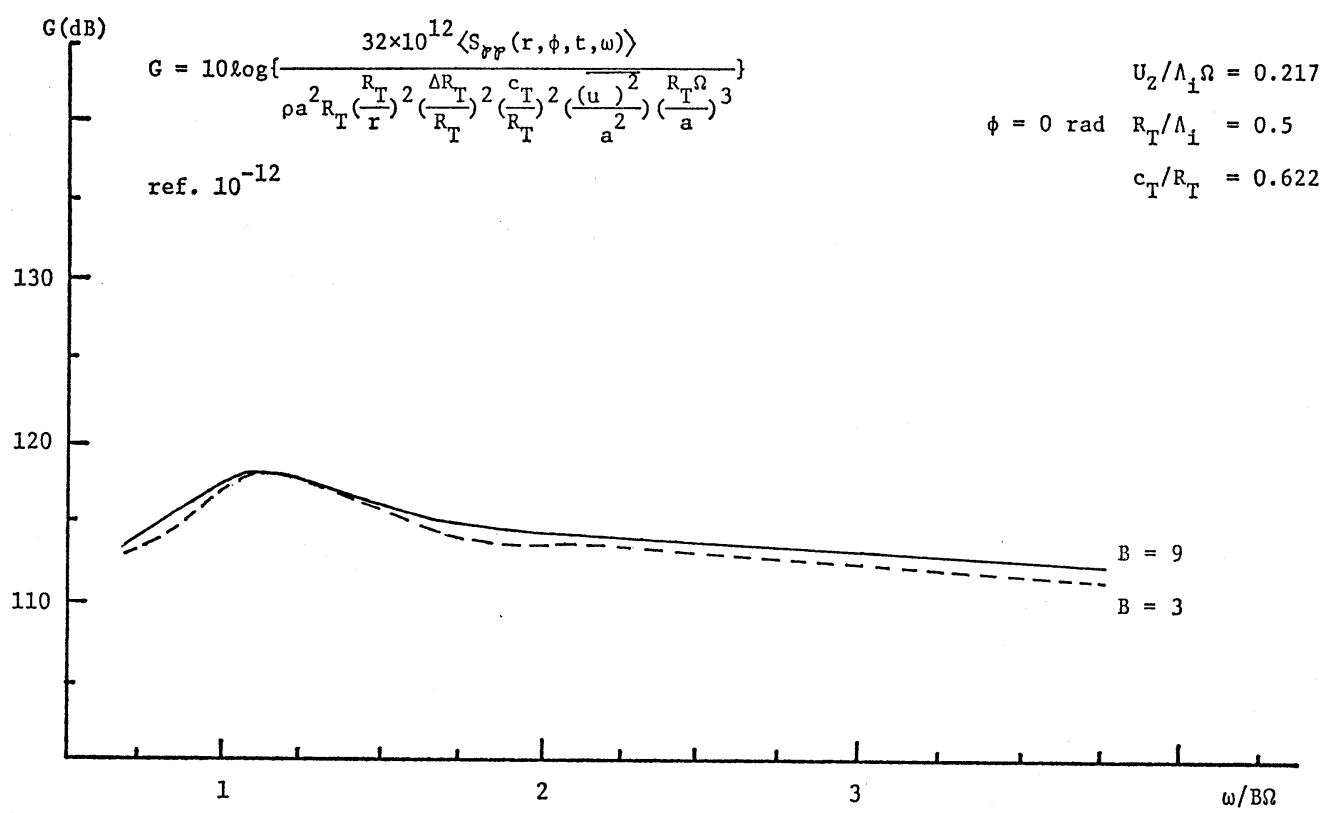

Fig. 7 Blade number dependence of non-dimensionalized $\left\langle S_{\mathscr{Q} \mathscr{Q}}(r, \phi, t, \omega)\right\rangle$ for $U_{z} / \Lambda_{\mathrm{i}} \Omega=$ 0.217 and $R_{\mathrm{T}} / \Lambda_{\mathrm{i}}=0.5$.

of the non-dimensionalized $\left\langle S_{\mathscr{Q} \mathscr{P}}(r, \phi, t, \omega)\right\rangle$. As shown in this figure, the amplitude modulation terms due to propagation path variation, i.e., $e_{\mathrm{T}}^{(p)}$ and $f_{T}^{(p)}$ in Eq. (3.8), gradually influence the spectral profile as the observer angle increases. Thus, the domination of the blade-passing frequency is reduced as the observer angle increases. 


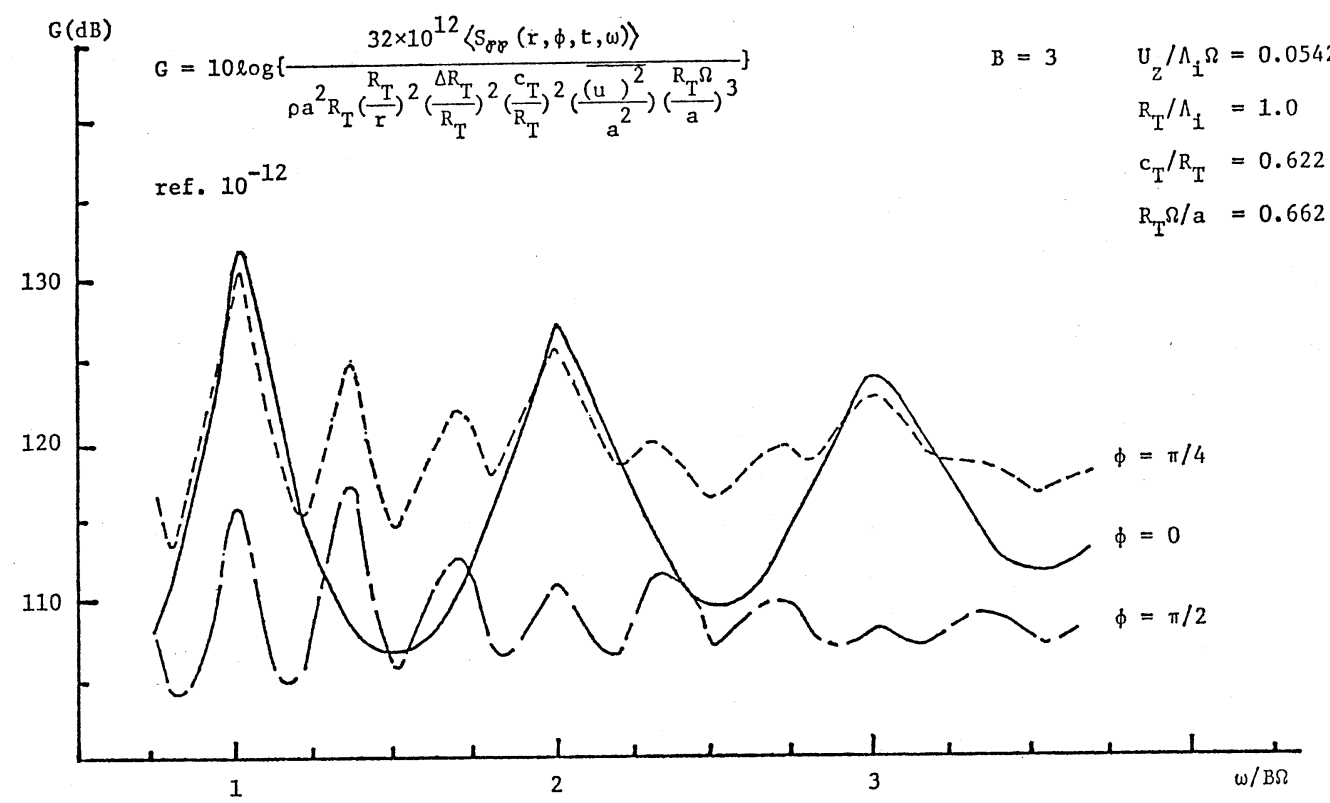

Fig. $8 \phi$-Dependence of non-dimensionalized $\left\langle S_{\mathscr{Q} \mathcal{Q}}(r, \phi, t, \omega)\right\rangle$ for $B=3, U_{z} / \Lambda_{\mathrm{i}} \Omega=0.0542$, and $R_{\mathrm{T}} / \Lambda_{\mathrm{i}}=1.0$.

\section{CONCLUSION}

The time-averaged intensity spectral density function of the acoustic radiation from rotating blades was derived by replacing blades with rotating dipoles. The following conclusions can be drawn from the derivation and the numerical calculation:

1) The rectilinear model of a rotating blade row is applicable only for the frequency range, $\omega \gg \Omega$ for which the 2nd term in Eq. (2.5) can be neglected compared with other terms.

2) The effect of the variation of the distance between the observer and rotating dipoles can be neglected when $R_{\mathrm{T}} \Omega \sin \phi / a \ll 1$. However, even when $\phi=0$, the blade-passing frequency and its multiples appear according to the inflow condition; see Fig. 8 and Eq. (3.8).

3) Two non-dimensional parameters, $U_{z} / \Lambda_{\mathrm{i}} \Omega$ and $R_{\mathrm{T}} / \Lambda_{\mathrm{i}}$, govern the profile of the noise spectrum. As $U_{z} / \Lambda_{\mathrm{i}} \Omega$ decreases, the blade-passing frequency and its multiples appear clearly, and as $R_{\mathrm{T}} / \Lambda_{\mathrm{i}}$ increases they do also; see Figs. 4, 5, and 6.

4) The non-dimensionalized time average acoustic intensity density function is proportional to $\left(\overline{u^{2}} / a^{2}\right)\left(R_{\mathrm{T}} / a\right)^{3}$ under the condition that other nondimensional parameters are fixed; Eq. (3.8).
5) The acoustic intensity at the off-angle from the rotor axis is not statistically stationary even if the inflow turbulence is frozen and homogeneous; see Eq. (2.5).

6) Increasing $B$, and/or increasing $\Omega$ under the condition that $U_{z} / \Lambda_{\mathrm{i}} \Omega$ is fixed increases the bandwidth of the peak of the blade-passing frequency and its multiples; see Fig. 7.

\section{ACKNOWLEDGEMENTS}

The author greatly acknowledges Dr. D.E. Thompson, who suggested the topic. His guidance will awalys be remembered.

The author acknowledges the support of Applied Research Laboratory, The Pennsylvania State University, under contract with the U.S. Naval Sea Systems Command.

\section{REFERENCES}

1) N. H. Kemp and W. R. Sears, "Aerodynamic interference between moving blade rows," J. Aeronaut. Sci. 20, 585-597 (1953).

2) M.V. Lowson, "Sound field for singularities in motion," Proc. R. Soc. London Ser. A286, 559-572(1956).

3) M. M. Sevik, "The response of a propulsor to random velocity fluctuations," ORL Serial No. N00017-70-C-1407-2 (1970). 


\section{K. ISHIMARU: NOISE SPECTRUM FROM ROTATING BLADES}

4) B. Robbins and B. Lakshminarayana, "Effect of inlet turbulence on compressor noise," J. Aircr. 11, 273-281 (1974).

5) F. Farassat,' "Theory of noise genaration from moving bodies with an application to helicopter rotors," NASA TR-451 (1975).

6) C. L. Morfey and H. K. Tanna, "Sound radiation from a point force in circular motion," J. Sound Vib. 15, 325-351 (1971).

7) H. W. Liepmann, "On the application of statistical concepts to the buffeting problem," J. Aeronaut. Sci. 19, 793-800 (1952).

8) J. P. Giesing, W. P. Rodden, and B. Stahl, "Sears function and lifting surface theory for harmonic gusts," J. Aircr. 7, 252-255 (1970).

9) F. Lane, "Broadband noise generated by turbulent inflow to rotor or stator blades in an annular duct," NASA TR-4 (1975).

10) R. Jackson, J. M. R. Graham, and D. J. Maull, "The lift on a wing in a turbulent flow," Aeronaut. Q. 24, 155-166 (1973).

11) R. K. Amiet, "Acoustic radiation from an airfoil in a turbulent flow," J. Sound Vib. 41, 407-420 (1975).

12) R. Mani, "Noise due to interaction of inlet turbulence with isolated stators and rotors," J. Sound Vib. 17, 251-260 (1971).

13) G. F. Homicz, "Broadband and discrete frequency noise radiation from subsonic rotors," $\mathrm{Ph}$. D. Thesis, Cornell Univ. (1973).

\section{NOMENCLATURE}

$a$ : $\quad$ speed of sound $[\mathrm{m} / \mathrm{s}]$

$B$ : number of blades

$c_{T}$ : chord length of typical segment of blade $[\mathrm{m}]$

$D_{m n}$ : distance between observer between $n$th segment of $m$ th blade

$e_{m n}{ }^{(p)}:$ defined in Eq. (2.6)

$f_{m n}{ }^{(p)}$ : defined in Eq. (2.7)

$\vec{f}:$ body force defined in Eq. (2.3) [N/ $\left./ \mathrm{m}^{3}\right]$

$H(\omega)$ : transfer function of typical segment of blade

$k$ : integer

$\vec{l}_{m n}(\tau):$ lift per unit span on $n$th segment of $m$ th blade $[\mathrm{N} / \mathrm{m}]$

$l_{m n}(\tau):$ modular of $\vec{l}_{m n}(\tau)[\mathrm{N} / \mathrm{m}]$

$m$ : integer

$N:$ number of segments

$p$ : harmonic number

$\mathscr{L}(r, \phi, t):$ pressure at observer $\left[\mathrm{N} / \mathrm{m}^{2}\right]$

$q$ : harmonic number

$r, \theta, \phi:$ spherical coordinates for observer

$R_{\mathrm{T}}$ : radial position of typical segment of blade

$R, \Phi, Z:$ cylindrical coordinates for rotor

$\Delta R_{\mathrm{T}}$ : length of typical segment of blade

$\mathcal{R}_{m k}^{\mathrm{uu}}(\alpha)$ : correlation function of upwash between $m$ th blade and $k$ th blade

$S_{m k}^{\mathrm{uu}}(\omega)$ : Fourier transform of $\mathcal{R}_{m k}^{\mathrm{uu}}(\alpha)$

$t$ : time associated with observer [s]

$\overline{u^{2}}$ : mean square value of velocity fluctuation of inflow turbulence

$U_{z}:$ axial flow velocity $[\mathrm{m} / \mathrm{s}]$

$\alpha:$ time difference

$\beta_{m n}$ : stagger angle at $n$th segment of $m$ th blade

$\beta_{\mathrm{T}}$ : stagger angle at typical segment of blade

$\delta(x)$ : Dirac delta function

$\delta_{s 0}$ : Kronecker delta where $s$ is zero

$\omega$ : angular frequency associated with sound

$\Omega:$ rotational speed of blade $[\mathrm{rad} / \mathrm{s}]$

$\rho$ : density

$\Lambda_{\mathrm{i}}$ : integral length scale $[\mathrm{m}]$

$\tau$ : time associated with noise source

$\Phi_{m}: 2 \pi m / B$

\langle\rangle : time average over $2 \pi / \Omega$ [s]

Superscripts

$(p)$ : harmonic number

$(q)$ : harmonic number

u: upwash of blade

*: complex conjugate

Subscripts

$B: \quad B$ rotor blades

$k: k$ th blade

$m$ : $m$ th blade

$n: \quad n$th blade

$\mathscr{Q}$ : acoustic pressure

$\mathrm{T}$ : typical segment

\section{APPENDIX}

Lowson $^{2)}$ derived the following Fourier coefficients of the amplitude modulation for $3 \mathrm{rd}$ and 4th terms in Eq. (2.4), under the condition $\Phi_{m}=0$,

$$
\begin{aligned}
& \frac{\cos \Omega\left(t-D_{m n} / a\right)}{\left(1+\frac{R_{n} \Omega}{a} \sin \phi \sin \Omega\left(t-D_{m n} / a\right)\right)^{3}} \\
& =\sum_{p=-\infty}^{\infty} \frac{-j p J_{p}\left(p \frac{R_{n} \Omega}{a} \sin \phi\right)}{\frac{R_{n} \Omega}{a} \sin \phi} e^{j p(\Omega t+\pi / 2)} .
\end{aligned}
$$


Taking the phase $\Phi_{m}$, of the amplitude modulation in Eq. (2.4) into account yields

$$
\begin{gathered}
\frac{\cos \left\{\Omega\left(t-D_{m n} / a\right)+\Phi_{m}\right\}}{\left(1+\frac{R_{n} \Omega}{a} \sin \phi \sin \left\{\Omega\left(t-D_{m n} / a\right)+\Phi_{m}\right\}\right)^{3}} \\
=\sum_{p=-\infty}^{\infty} \frac{-j p J_{p}\left(p \frac{R_{n} \Omega}{a} \sin \phi\right)}{\frac{R_{n} \Omega}{a} \sin \phi} e^{j p\left(\Omega t+\pi / 2+\Phi_{m}\right)}
\end{gathered}
$$

(A.2)

Now, consider the Fourier coefficient of the other amplitude modulation in Eq. (2.4), for instance, the Fourier coefficient of $\left(1+\left(R_{n} \Omega / a\right) \sin \phi \sin \{\Omega(t-\right.$ $\left.\left.D_{m n}(a)+\Phi_{m}\right\}\right)^{-2}$. Taking the time derivative of $\left(1+\left(R_{n} \Omega / a\right) \sin \phi \sin \left\{\Omega\left(t-D_{m n} / a\right)+\Phi_{m}\right\}\right)^{-1}$ yields

$$
\begin{gathered}
\frac{d}{d t}\left\{1+\left(R_{n} \Omega / a\right) \sin \phi \sin \gamma\right\}^{-1} \\
=-\frac{\Omega^{2} R_{n} \sin \phi \sin \gamma}{a\left(1+\frac{R_{n} \Omega}{a} \sin \phi \sin \gamma\right)^{3}},
\end{gathered}
$$

where the following relations are used:

$$
\begin{gathered}
\frac{d}{d t}=\frac{d \gamma}{d T} \frac{d T}{d t} \frac{d}{d \gamma}, \\
\gamma=\Omega\left(t-D_{m n} / a\right)+\Phi_{m},
\end{gathered}
$$

and

$$
T=\Omega(t-r / a)+\Phi_{m}=\gamma-\frac{R_{n} \Omega}{a} \sin \phi \cos \gamma .
$$

By comparing Eq. (A.2) with Eq. (A.3), one obtains the following expansion:

$$
\begin{aligned}
(1 & \left.+\frac{R_{n} \Omega}{a} \sin \phi \sin \left\{\Omega\left(t-D_{m n} / a\right)+\Phi_{m}\right\}\right)^{-1} \\
= & \sum_{p=-\infty}^{\infty} J_{p}\left(p \frac{R_{n} \Omega}{a} \sin \phi\right) e^{j p\left(\Omega t+\pi / 2+\Phi_{m}\right)},
\end{aligned}
$$

where the steady state component can be computed by

$$
\frac{\Omega}{2 \pi} \int_{0}^{2 \pi / \Omega}\left(1+\frac{R_{n} \Omega}{a} \sin \phi \sin \gamma\right)^{-1} d t=1,
$$

since $d \gamma=\left(1+\frac{R_{n} \Omega}{a} \sin \phi \sin \gamma\right)^{-1} d t$.

Therefore, one obtains

$$
\begin{aligned}
(1+ & \left.\frac{R_{n} \Omega}{a} \sin \phi \sin \left\{\Omega\left(t-D_{m n} / a\right)+\Phi_{m}\right\}\right)^{-2} \\
= & \sum_{p=-\infty}^{\infty} \sum_{q=-\infty}^{\infty} J_{p}\left(p \frac{R_{n} \Omega}{a} \sin \phi\right) J_{q}\left(q \frac{R_{n} \Omega}{a} \sin \phi\right) \\
& \times e^{j(p+q)\left(\Omega t+\pi / 2+\Phi_{m}\right)} .
\end{aligned}
$$

Now, consider the Fourier coefficient of $\sin \{\Omega(t$ $\left.\left.-D_{m n} / a\right)+\Phi_{m}\right\} /\left(1+\left(R_{n} \Omega\right) / a\right) \sin \phi \sin \left\{\Omega\left(t-D_{m n} / a\right)\right.$ $\left.\left.+\Phi_{m}\right\}\right)^{2}$. Since it can de expressed by

$$
\begin{gathered}
\frac{\sin \left\{\Omega\left(t-D_{m n} / a\right)+\Phi_{m}\right\}}{1+\frac{R_{n} \Omega}{a} \sin \phi \sin \left\{\Omega\left(t-D_{m n} / a\right)+\Phi_{m}\right\}} \\
\cdot \frac{1}{1+\frac{R_{n} \Omega}{a} \sin \phi \sin \left\{\Omega\left(t-D_{m n} / a\right)+\Phi_{m}\right\}},
\end{gathered}
$$

the Fourier coefficient can be obtained through coefficients for each term in the above.

Taking the time derivative of $\sin \left\{\Omega\left(t-D_{m n} / a\right)+\right.$ $\left.\Phi_{m}\right\} /\left(1+\left(R_{n} \Omega / a\right) \sin \phi \sin \left\{\Omega\left(t-D_{m n} / a\right)+\Phi_{m}\right\}\right)$ yields

$$
\begin{aligned}
& \frac{d}{d t}\left(\frac{\sin \gamma}{1+\frac{R_{n} \Omega}{a} \sin \phi \sin \gamma}\right) \\
& =\frac{\Omega \cos \gamma}{\left(1+\frac{R_{n} \Omega}{a} \sin \phi \sin \gamma\right)^{3}},
\end{aligned}
$$

where Eq. (A.4), Eq. (A.5), and Eq. (A.6) are utilized.

By comparing Eq. (A.10) with Eq. (A.2), the following expansion can be obtained:

$$
\begin{aligned}
& \frac{\sin \gamma}{1+\frac{R_{n} \Omega}{a} \sin \phi \sin \gamma} \\
& =\sum_{p=-\infty}^{\infty}-\frac{J_{p}\left(p \frac{R_{n} \Omega}{a} \sin \phi\right)}{\frac{R_{n} \Omega}{a} \sin \phi} e^{j p\left(\Omega t+\pi / 2+\Phi_{m}\right)}\left(\delta_{0 p}-1\right),
\end{aligned}
$$

where the steady state component is obtained by

$$
\frac{\Omega}{2 \pi} \int_{0}^{2 \pi / \Omega} \frac{\sin \gamma}{1+\frac{R_{n} \Omega}{a} \sin \phi \sin \gamma} d t=\frac{1}{2 \pi} \int_{0}^{2 \pi} \sin \gamma d \gamma
$$$$
=0 \text {. }
$$

Therefore, utilizing Eq. (A.9) and Eq. (A.12), the following expansion is obtained:

$$
\begin{gathered}
\frac{\sin \left\{\Omega\left(t-\frac{D_{m n}}{a}\right)+\Phi_{m}\right\}}{\left(1+\frac{R_{n} \Omega}{a} \sin \phi \sin \left\{\Omega\left(t-\frac{D_{m n}}{a}\right)+\Phi_{m}\right\}\right)^{2}} \\
=\sum_{p=-\infty}^{\infty} \sum_{q=-\infty}^{\infty} \frac{\delta_{0 p}-1}{\frac{R_{n} \Omega}{a} \sin \phi} J_{p}\left(p \frac{R_{n} \Omega}{a} \sin \phi\right) \\
\times J_{q}\left(q \frac{R_{n} \Omega}{a} \sin \phi\right) e^{j(p+q)\left(\Omega t+\Phi_{m}+(\pi / 2)\right)} .
\end{gathered}
$$

